\title{
Les Syndicats irlandais et la question cubaine, 50 ans après la révolution
}

Irish Trade Unions \& the Cuban Connection: 50 years after the Cuban revolution

\section{Marie-Violaine Louvet}

\section{(2) OpenEdition}

12 Journals

\section{Édition électronique}

URL : http://journals.openedition.org/etudesirlandaises/6073

DOI : $10.4000 /$ etudesirlandaises. 6073

ISSN : 2259-8863

\section{Éditeur}

Presses universitaires de Caen

\section{Édition imprimée}

Date de publication : 18 décembre 2018

Pagination : $37-52$

ISBN : 978-2-7535-7693-3

ISSN : 0183-973X

\section{Référence électronique}

Marie-Violaine Louvet, "Les Syndicats irlandais et la question cubaine, 50 ans après la révolution », Études irlandaises [En ligne], 43-2 | 2018, mis en ligne le 01 novembre 2018, consulté le 13 septembre 2019. URL : http://journals.openedition.org/etudesirlandaises/6073; DOI : 10.4000/ etudesirlandaises.6073 


\title{
Les syndicats irlandais et la question cubaine, 50 ans après la révolution
}

\author{
Marie-Violaine LouveT \\ Université Toulouse 1-Capitole
}

\section{Résumé}

En 2009, les festivités autour du cinquantenaire de la révolution cubaine sont l'occasion pour les syndicats irlandais de réaffirmer leur attachement au régime socialiste de Fidel Castro. Dans leur discours, qui mêle phantasmes révolutionnaires et références historiques, le mythe d'un Cuba rebelle et socialiste vient flirter avec l'imagerie de l'anti-impérialisme irlandais alors que les campagnes contre l'embargo américain et pour la libération des cinq de Miami trouvent un second souffle.

Mots clés : Cuba, Irlande, syndicats, révolution, Fidel Castro, embargo, cinq de Miami, ICTU, SIPTU

\section{Abstract}

In 2009, the celebrations surrounding the fiftieth anniversary of the Cuban revolution were an opportunity for Irish trade unions to explore their connections with the socialist regime of Fidel Castro. Their discourse, punctuated by revolutionary phantasies and historical references, mixed the myth of the rebellious Cuba with the imagery of Irish anti-imperialism. The anniversary gave momentum to their campaigns against the American embargo on Cuba and for the liberation of the Cuban five. SIPTU

Keywords: Cuba, Ireland, trade unions, revolution, Fidel Castro, blockade, Cuban five, ICTU,

En République d'Irlande, l'engouement autour de la vente de timbres à l'effigie du Che Guevara, dont on fêtait en 2017 le 50 anniversaire de la mort, révéla une certaine fascination populaire pour la figure socialiste ${ }^{1}$. C'est la célèbre image du révolutionnaire, créée par l'artiste irlandais Jim Fitzpatrick à partir d'une photo prise par Alberto Korda en 1960, qui illustrait cette collection unique de timbres. Le Che, décrit par An Post, le service postal irlandais, comme "la quintessence du révolutionnaire de gauche ${ }^{2}$ ", avait un ancêtre irlandais, Patrick Lynch, né à

1. Les 122000 exemplaires de la première impression ont tous été vendus très rapidement, voir " First print of controversial Che Guevara stamp sells out ", The Irish Times, 12 octobre 2017.

2. Les termes utilisés par An Post sont " the quintessential left-wing revolutionary ", voir le site internet $d^{\prime} A n$ 
Galway en 1715, raison pour laquelle Ernesto Guevara Lynch - le père du Che affirma que le sang des rebelles irlandais coulait dans les veines de son fils ${ }^{3}$.

La figure du Che, est indissociable de celle de Fidel Castro, qui dirigea Cuba de la fin de la révolution (1959), jusqu'à quelques années avant son décès, le 25 novembre 20164. Le Président irlandais Michael D. Higgins - lui-même membre du Parti travailliste pendant 40 ans - fut le premier officiel à signer le livre de condoléances ouvert à l'ambassade cubaine. À cette occasion, il affirma que «... [L]a vision de Castro n’était pas seulement une vision de liberté pour son peuple mais pour tous les peuples opprimés et exclus de la planète ". Son commentaire élogieux provoqua des protestations véhémentes, notamment de la part de Cubains exilés aux États-Unis, qui soulignèrent la répression sévère menée à l'encontre des opposants politiques par le régime castriste.

La popularité de ces figures de la révolution cubaine est remarquable au sein des cercles politiques influencés par le socialisme, notamment le mouvement syndical irlandais. On le voit en particulier en 2009, année du cinquantenaire de la révolution cubaine de 1959. En effet, à l'occasion de cet anniversaire, la fédération nationale de syndicats, l'Irish Congress of Trade Unions (ICTU), tout comme certains de ses syndicats affiliés à titre individuel, fut à l'origine de multiples actions qui témoignèrent de leur admiration pour l'héritage de la révolution menée par Fidel Castro ${ }^{6}$. L’année 2009, qui correspondit aussi au cinquantenaire de la création de l'Irish Congress of Trade Unions ${ }^{7}$, fut un moment privilégié qui marqua un accroissement des échanges entre les syndicats irlandais et différents groupes cubains.

La question de l'appropriation de la question cubaine par les syndicats irlandais, et des liens de collaboration qui sont tissés avec la société civile et le gouvernement cubain, est à ce jour inexplorée dans la littérature scientifique, contrai-

Post, "Iconic Che gets stamp treatment ", 9 octobre 2017, [http://www.anpost.ie/AnPost/MainContent/About+An+Post/Media+Centre/Press+Releases/2017/Iconic+Che+gets+stamp+treatment.htm?wbc_ purpose=Basic], consulté le 27 février 2018.

3. Les termes précis sont "in my son's veins flowed the blood of Irish rebels». Ils figurent sur une enveloppe vendue par An Post en complément des timbres. Voir Vivienne Clarke, Fiach Kelly et Ronan McGreevy, "What is Ireland doing putting Che Guevara on a stamp? ", The Irish Times, 9 octobre 2017.

4. Fidel Castro fut Premier ministre de Cuba de 1959 à 1976, Président de Cuba de 1976 à 2008, et premier secrétaire du parti communiste cubain de 1961 à 2011.

5. Les termes exacts sont: «Fidel Castro will be remembered as a giant among global leaders whose view was not only one of freedom for his people but for all of the oppressed and excluded peoples on the planet ", voir Ian Begley, "A giant among global leaders' - Irish President leads tributes to Fidel Castro ", The Irish Independent, 26 novembre 2016. Par ailleurs Michael D. Higgins se rendit en visite officielle à Cuba, en février 2017.

6. Voir "Seizing the moment », le discours de Patricia McKeown, Présidente de l'ICTU, à la conférence bisannuelle du Congrès à Tralee en 2009, disponible sur le site internet de l'ICTU, [https://www.ictu.ie/press/2009/07/07/ seizing-the-moment-patricia-mckeown-ictu-president/], consulté le 27 février 2018.

7. L'Irish Congress of Trade Unions naît de la fusion de l'Irish Trade Union Congress et du Congress of Irish Unions en 1959. 
rement à la lutte syndicale contre l'apartheid en Afrique du Sud, dans les années 1980. Les causes internationales défendues par les syndicats irlandais au $20^{\mathrm{e}}$ siècle ne sont pas très nombreuses, et se concentrent principalement sur la lutte pour la fin de l'apartheid en Afrique du Sud, et le soutien à la cause palestinienne ces vingt dernières années. Le sujet cubain n'est pas aussi consensuel que ne l'était celui de la lutte contre l'apartheid à cause de la nature du régime politique qui règne sur l'île. Afin d'explorer cette thématique cubaine au sein du mouvement syndical irlandais, cet article repose sur l'étude de la littérature grise publiée à la fois par la fédération nationale des syndicats irlandais, l'ICTU, et les différents syndicats qui lui sont affiliés : rapports des délégations qui visitèrent Cuba en 2009, prospectus de propagande, fiches d'information, magazines à destination des membres, ouvrages publiés pour usage interne, sites internet, réseaux sociaux - mais aussi communiqués de presse, et discours à destination du grand public.

À partir de ces sources primaires, cet article se propose d'analyser les mobilisations syndicales irlandaises autour du cinquantenaire de la révolution cubaine en 2009, afin de mettre en lumière la persistance d'une mythologie révolutionnaire qui peine à se confronter à la réalité du castrisme aujourd'hui, à l'épreuve des contacts directs avec la société civile cubaine. Les résonnances, toutes particulières en Irlande, du discours pro-cubain, fondé sur les notions d'anti-colonialisme et d'anti-impérialisme seront d'abord étudiées, à travers l'identification de mythes qui viennent lier les histoires irlandaises et cubaines. Puis l'action militante engagée dans les deux principales campagnes ralliées par les syndicats irlandais sera analysée, avec d'abord la protestation contre l'embargo américain sur l'île de Cuba et ensuite l'appel à la libération des « cinq de Miami ${ }^{8}$ ».

\section{Création d'une mythologie cubaine dans le discours syndical en Irlande}

Pour les syndicats irlandais, s'emparer du récit de la révolution cubaine de la fin des années 1950, c'est parler de leurs propres aspirations et de leurs propres valeurs dans l'Irlande d'aujourd'hui. En termes de mythe, l'histoire cubaine dans son ensemble est intéressante car elle comporte des figures héroïques, qui sont engagées dans un paradigme de la résistance à un ennemi incommensurablement plus puissant - la lutte contre l'impérialisme espagnol à la fin du XIX ${ }^{e}$ siècle, puis contre les États-Unis, alliés du gouvernement de Fulgencio Batista au $\mathrm{xx}^{\mathrm{e}}$ siècle?. Le gouver-

8. «Cuban Five » ou « les Cinq de Miami » est l'expression consacrée qui désigne cinq citoyens cubains arrêtés par les autorités américaines à Miami en 1998. Pour plus de détails, voir la section «Les cinq de Miami : Gerardo Hernández, Ramón Labañino, Fernando González, Antonio Guerrero, René González », de cet article.

9. Fulgencio Batista, qui fut Président de Cuba entre 1940 et 1944, s'empara du pouvoir par la force en 1952 et mena le pays d'une main de fer jusqu'à la révolution de 1959. Entre 1944 et 1952, il vécut en Floride et il 
nement cubain lui-même a une maîtrise certaine de la communication au sujet de son histoire révolutionnaire, comme on peut le voir avec le magnifique Museo de la Revolución, à La Havane, qui retrace l'histoire de la révolution de 1959 contre Batista mais aussi de la guerre d'indépendance contre l'empire espagnol (18961898). En 2009, lors du cinquantenaire de la révolution cubaine, deux figures rapprochant l'histoire irlandaise de l'histoire cubaine sont commémorées par l'ICTU et SIPTU ${ }^{10}$ : José Martí (1853-1895) et James Joseph O’Kelly (1845-1916).

\section{José Martí et James Joseph O'Kelly : traits d'union entre Cuba et l'Irlande}

José Martí et James Joseph O'Kelly, deux personnages historiques engagés dans la dénonciation des crimes de l'empire espagnol lors de la guerre d'indépendance de Cuba, ont ceci en commun qu'ils furent un trait d'union entre Cuba et l'Irlande à la fin du XIx siècle. Il s'agit alors d'un moment charnière de l'histoire de l'île de Cuba mais aussi d'une Irlande défigurée par les affres de la grande famine (1845-1852) et agitée par la campagne en faveur de plus d'autonomie vis-à-vis de Londres ${ }^{11}$.

Il convient tout d'abord d'évoquer le héros national cubain José Martí, dont le nom fut fréquemment cité dans le discours des syndicats irlandais sur Cuba en 2009. José Martí fut avant tout un intellectuel et un écrivain, qui, épris de l'action politique et "apôtre d'un Cuba indépendant ", mourut sur le champ de bataille pendant la guerre d'indépendance de Cuba contre l'Espagne ${ }^{12}$. Dans ses chroniques, écrites en exil aux États-Unis entre juillet 1881 et mai 1892, Martí étudia et commenta la vie de la diaspora irlandaise aux États-Unis ${ }^{13}$. Comme l'a montré Felix Flores Varona, il développa alors un intérêt tout particulier pour les grandes figures politiques irlandaises que furent Daniel O'Connell, Michael Davitt et Charles Stewart Parnell ${ }^{14}$. Micheál Martin ${ }^{15}$, ancien Ministre des Affaires étrangères de la République d'Irlande, effectua la première visite officielle d'un ministre

bénéficia, pendant ses mandats, du soutien américain.

10. SIPTU (Services, Industrial, Professional and Technical) est un syndicat irlandais qui compte plus de 200000 adhérents.

11. On fait ici référence au "Home Rule movement", ce mouvement qui domine le nationalisme irlandais dans le dernier quart du $19^{\mathrm{e}}$ siècle et qui demande une autonomie gouvernementale pour l'Irlande au sein du Royaume-Uni.

12. José Martí est souvent désigné par les termes affectueux de "apóstolo de la independiencia » à Cuba.

13. Les chroniques de Martí furent publiées dans divers journaux d'Amérique latine tels que La Opinión Nacional du Vénézuela, La Nación d'Argentine, El Partido Liberal du Mexique ou encore La República, du Honduras.

14. Papier intitulé "The Irish element in José Marti’s New York Chronicles », Félix Flores Varona (Universidad de Ciego de Ávila), présenté les 27-30 juin 2007, à National University of Ireland, Galway, [http://www.irlandeses.org/galway_varona.htm], consulté le 27 février 2018.

15. Michéal Martin appartient au parti Fianna Fáil, centre-droit, dans la République d'Irlande. Le Fianna Fáil se désigne comme un parti républicain, engagé pour l'unité de lì̂le d'Irlande en une seule entité politique. Voir le site internet de Fianna Fáil, [https://www.fiannafail.ie/about-fianna-fail/], consulté le 27 février 2018. 
irlandais à Cuba en février 2009, et alla lui-même déposer une couronne sur le mausolée en l'honneur de José Martí, qui se trouve sur la Plaza de la Revolución, à La Havane ${ }^{16}$.

Si l'on érigeait un panthéon des personnalités qui servirent de relais entre l'Irlande et Cuba, James Joseph O'Kelly figurerait en bonne place. C'est SIPTU qui le mit particulièrement à l'honneur en 2009, année qui marqua à la fois le centenaire de la création de SIPTU et les cinquante ans de la révolution cubaine ${ }^{17}$. En effet, SIPTU publia l'ouvrage Irish Solidarity with Cuba Libre: a Fenian eyewitness account of the first Cuban war of independence ${ }^{18}$, édité par Manus O'Riordan, membre de SIPTU mais aussi chargé de la recherche pour cette formation syndicale $^{19}$. Manus O'Riordan est le fils de Micheál O’Riordan, membre de l'Irish Transport and General Workers Union (ITGWU) ${ }^{20}$ et l'un des fondateurs du parti communiste d'Irlande ${ }^{21}$. Par ailleurs, SIPTU souligna que c'est grâce à deux pionniers de l'ITGWU, William O'Brien et Frank Robbins - respectivement ancien secrétaire général et ancien secrétaire pour la région de Dublin ${ }^{22}$ - que les manuscrits de O’Kelly purent être archivés ${ }^{23}$. O’Kelly était une figure du mouvement fénien, membre du parlement britannique qui défendait plus d'autonomie pour l'Irlande, et correspondant pour le New York Herald pendant la première guerre d'indépendance de Cuba contre l'Espagne. Il décrivit cette guerre comme une lutte de libération nationale, dépeignant avec verve la "terrible sévérité du gouvernement espagnol " à l'encontre de la population cubaine ${ }^{24}$. Michael Davitt lui rendit par ailleurs hommage dans The Fall of Feudalism in Ireland, publié en $1904^{25}$. O’Kelly fut enterré au cimetière de Glasnevin, à Dublin, auprès de héros

16. John Moran, «A changed landscape as Cuba marks revolution ", The Irish Times, 18 février 2009.

17. Plus précisément de l'Irish Transport and General Workers' Union, créé en 1909, dont la fusion avec Federated Workers' Union of Ireland donna naissance à SIPTU en 1990.

18. Manus O'Riordan, Irish Solidarity with Cuba Libre: a Fenian eyewitness account of the first Cuban war of independence, Dublin, SIPTU, 2009.

19. "Head of Research " est le titre précis.

20. L'Irish Transport and General Workers Union fut fondé en 1909 par Jim Larkin. De sa fusion avec Federated Workers' Union of Ireland, en 1990, naquit SIPTU.

21. Le parti communiste d'Irlande fut fondé en 1933.

22. Les titres exacts sont "General Secretary " pour William O’Brien et "Dublin District Council Secretary " pour Frank Robbins.

23. Manus O'Riordan, Irish Solidarity with Cuba Libre: a Fenian eyewitness account of the first Cuban war of independence, Dublin, SIPTU, 2009, introduction.

24. L'expression en anglais est "terrible severity of the Spanish government », ibid., p.69. Les chiffres varient, mais on estime à plus de 100000 le nombre de civils cubains qui moururent pendant cette guerre, en particulier suite à un internement dans des camps dans des conditions déplorables, voir John Lawrence Tone, War and Genocide in Cuba, 1895-1898, Chapel Hill, The University of North Carolina Press, 2006, p. 210-212.

25. "Mr. James O'Kelly's name must be honorably associated with those mentioned in connection with this early and valuable labor. He has always been a broad-minded thinker and worker in national movements. Narrow views or petty prejudices were never peculiar to Mr. O'Kelly. He favored every kind of useful action that could advance a cause in the service of which he has given the best years of his life, and throughout his varied and romantic career he has preferred the part of a silent worker to that which earns most distinction by inviting most public notice. " Dans 
de l'histoire irlandaise, tels que Daniel O'Connell et Charles Stewart Parnell. SIPTU lui organisa une cérémonie d'hommage en 2009, en présence de l'ambassadeur cubain.

Pour le cinquantenaire de la révolution cubaine, l'appropriation de l'histoire de Cuba par les syndicats irlandais comprit, nous l'avons vu, l'intermédiaire de figures contemporaines de la guerre d'indépendance qui rapprochèrent Cuba de l'Irlande, par le biais de leur sensibilité anti-impérialiste. La mise en valeur de ce faisceau idéologique commun, fut aussi caractéristique des commentaires des syndicalistes irlandais sur l'héritage de la révolution de 1959.

\section{Admiration pour certaines caractéristiques du régime socialiste cubain}

La mort de Castro fut l'occasion pour les syndicats irlandais de rendre hommage au Líder Máximo. Un événement intitulé "Farewell to Fidel» fut organisé en avril 2017 à Liberty Hall, le quartier général de SIPTU et le siège historique de l'ITGWU. Jack O'Connor, le Président Général de SIPTU ${ }^{26}$, Hermes Herrera Hernández, ambassadeur de Cuba en République d'Irlande ${ }^{27}$ et Jack McGinley, président de SIPTU Solidarity Forum with $C u b a^{28}$, se succédèrent pour évoquer les succès de Castro et fustiger la politique américaine à l'encontre de $\mathrm{Cuba}^{29}$. Le coordinateur national de Mandate ${ }^{30}$, Brian Forbes, insista pour qu'un livre de condoléances soit placé à la mairie de Galway, afin que les habitants de la ville puissent y laisser un message à la mémoire de Castro et à la gloire de son héritage :

That is Fidel Castro's legacy - a legacy of putting his country and its people before the extreme excesses and wealth of the one per cent [...] it is a fine example for us all as we fight to achieve a better, fairer, way for our people here, on this island. [...] Given Ireland's colonial history of oppression and of denied human rights, surely the small act of opening a book of condolence in recognizing Fidel Castro for all his great achievements and also for his love of the underdog is the least we can do.

Michael Devitt, The Fall of Feudalism in Ireland or The story of the Land League revolution, Londres, Harper \& Brothers, 1904, p. 128.

26. Jack O'Connor est General President de SIPTU depuis 2003, et fut président de l'Irish Congress of Trade Unions de 2009 à 2011. Depuis 2017, il dirige le Parti travailliste de la République d'Irlande.

27. Hermes Herrera est l'ambassadeur de Cuba en République d'Irlande depuis 2014.

28. Le groupe de travail SIPTU Cuban Solidarity Forum fut créé en 2005 au sein de SIPTU, sous l'égide de Kieran Jack McGinley. Kieran Jack McGinley fut membre du conseil national exécutif (National Executive Council) de SIPTU, ainsi que vice-président de son comité sur l'éducation. Il est toujours le président du SIPTU Cuban Solidarity Forum.

29. The Independent, 28 novembre 2016.

30. Mandate (the retail, bar and administrative workers' union) est un syndicat majeur en Irlande, avec plus de 40000 membres. 
Le cinquantenaire de la révolution cubaine en 2009 fut aussi l'occasion pour I'ICTU, SITPU mais aussi NIPSA, Unite, UCU, GMB, Trademark et le Belfast Unemployed Centre d'envoyer des délégations à $\mathrm{Cuba}^{31}$. Dans le rapport qui fut rédigé à leur retour, les délégués se déclarèrent particulièrement favorables au régime castriste à trois égards : la promotion de l'égalité entre les sexes, le niveau d'alphabétisation et la qualité du système de santé. Ils soulignèrent notamment que plus de $70 \%$ du budget annuel de Cuba était consacré à ces deux derniers postes de dépense ${ }^{32}$.

En matière d'égalité entre les sexes, le rôle prépondérant des femmes dans les instances nationales de Cuba fut largement mis en avant dans le rapport publié après la visite de la délégation syndicale irlandaise en $2009^{33}$. Pauline Collins, membre du conseil exécutif de University and College Union (UCU), va même jusqu'à souligner l'absence d'impact négatif de la domination numérique des femmes en responsabilité dans l'enseignement secondaire et l'université sur la population masculine, inversant ainsi le paradigme auquel tout observateur européen est accoutumé :

I found it interesting that even though most school principals and university lecturers are now female, Cuban males have no problems achieving the same levels of education as females. Maybe this is because the system is geared to learning, something we could we learn a lot from ${ }^{34}$.

La délégation de 35 membres de SIPTU qui se rendit à Cuba en novembre 2009 passa une journée à observer les actions de l'association Federación de Mujeres Cubanas, fondée en 1960 par Vilam Espin, la femme de Raúl Castro ${ }^{35}$, visant à la promotion des droits des femmes à Cuba. En mars 2009, Nancy Coro Aguiar, secrétaire générale de la Federación de Mujeres Cubanas, participa au séminaire consacré à la question des femmes dans la récession économique en Irlande, organisé à Belfast par l'ICTU ${ }^{36}$. Ces rencontres témoignent de la dynamisation des échanges entre certains membres de la société civile cubaine et certains syndicalistes irlandais et nord-irlandais. La place des femmes à Cuba est régulièrement

31. Brenda Callaghan, " 50 years on and still on march! », The Union Post, juin 2009, p. 32.

32. SIPTU, " Report on visit to Cuba by the SIPTU Solidarity with Cuba Forum November 9th-18th, 2009 ", p. 6.

33. L'égalité hommes-femmes à Cuba est aussi soulignée par l'International Trade Union Confederation, voir par exemple ITUC, "The global gender pay gap ", février 2008, p. 50.

34. Brenda Callaghan, ibid., p. 32.

35. Raúl Castro est le frère de Fidel Castro, qui lui succéda en tant que président en 2008 et en tant que premier secrétaire du parti communiste en 2011.

36. ICTU, "Irish Congress of Trade Unions Joint Women's Committees Seminar: Making the case for a strong focus on Gender Equality and Workers' Rights in Recessionary Times report ", 2009, p. 3. L'ICTU est actif en Irlande du Nord comme dans la République. 
mise en avant dans les rapports de l'ICTU, notamment leur influence dans la vie politique cubaine ${ }^{37}$.

Le cinquantenaire de la révolution cubaine fut aussi l'occasion pour l'ICTU de louer le système médical cubain, deuxième grande réussite du régime castriste reconnue par les syndicalistes irlandais. En 2009, lors de la conférence bisannuelle de l'ICTU qui eut lieu à Tralee, le syndicat Dublin Council of Trade Unions défendit une motion permettant d'évaluer "the success of the Cuban medical model in order to see what lessons car be learnt in the context of the current crises besetting the Irish health system ${ }^{38}$ ". Il s'agissait en particulier de faire face aux mesures d'austérité budgétaire décidées par le gouvernement irlandais après la crise économique de 2008. La délégation de SIPTU qui se rendit à Cuba en 2009 relata, dans un rapport, la journée qu'elle consacra à la visite du centre médical Hogar Benjamin Moreno, à La Havane. Elle insista en particulier sur l'humanité des méthodes utilisées, malgré le manque de moyens. SIPTU effectua un don de $10000 €$ à destination de 131 centres de soins médico-psychologiques ${ }^{39}$. Ce dernier vint compléter la somme de $60000 €$ rassemblée par l'ICTU en 2009, afin d'aider l'île de Cuba à faire face aux conséquences de la série d'ouragans qui s'abattit sur elle en septembre $2008^{40}$.

Enfin, dans le domaine de l'éducation, troisième pilier et garant symbolique du progressisme du régime cubain, les délégués de SIPTU rencontrèrent le Sindicato Nacional de Trabajadores de la Educación, la Ciencia y el Deporte (SNTECD) lors du premier jour de leur visite en 2009. Le rapport rédigé à leur retour loue en particulier le taux de $70 \%$ d'accès à l'université pour la classe d'âge des 18-23 ans et l'attention apportée aux enfants souffrant de handicaps. La conclusion encourage une collaboration accrue dans le domaine de l'éducation entre la République d'Irlande et Cuba, en particulier avec l'objectif de s'inspirer de certaines réussites du système cubain : "Given the strategic position of the Cuban educational and health policies and their world class rating, we recommend that a series of bipartite

37. Un rapport de l'ICTU consacré à l'égalité des sexes souligne, par exemple, que 43,2\% des parlementaires à Cuba sont des femmes, voir ICTU, «Gender equality and Global Solidarity: Decent work and the Millennium Development Goals ", 2012, p. 18.

38. Motion 47, "47. Evaluation of Cuban Medical Model Congress calls on the Government to undertake a study to evaluate the success of the Cuban medical model in order to see what lessons can be learned in the context of the current crises besetting the Irish health system. Dublin Council of Trade Unions ", dans ICTU, "Building Solidarity: Biennial delegate conference report, Tralee 7-10 July, 2009 ", 2009, p. 32.

39. SIPTU, "Report on visit to Cuba by the SIPTU Solidarity with Cuba Forum November 9th-18th, 2009 ", 2009, p. 11-12. Le rapport de la conférence bisannuelle de l'ICTU de 2011 souligna que la fédération était entrée en contact avec le Department of Health and Children, afin qu'une étude du modèle de santé cubain soit lancée. Voir ICTU, " Report of the Executive Council, Biennial delegate conference, Killarney 4-6 July 2011 », p. 154.

40. ICTU, "Building Solidarity: Biennial delegate conference report, Tralee 7-10 July, 2009 », 2009, p. 137. Sur la question des dons internationaux suite aux ouragans qui frappèrent les caraïbes et la propagande américaine, voir la publication de l'ICTU : "Media 'buried Cuba story' ", The Union Post, avril 2010, p. 8. 
engagements take place including student, postgraduate and practicing medics placements in the other country so that we both might understand and learn from each other's systems ${ }^{41} "$.

Dans le récit de retour des syndicalistes irlandais qui visitèrent Cuba en 2009, l'égalité hommes-femmes, tout comme la qualité de l'éducation et du système de santé sont les pierres d'angle de l'utopie cubaine caractérisée par les valeurs progressistes qui régissent cette société. Pourtant, ce bilan, qui ne semble nuancé que par les difficultés matérielles que rencontre l'île, n'est pas similaire à celui qui est fait par certaines autres organisations de travailleurs en Europe et dans le monde.

\section{Contraste avec le discours de l'ETUC/ILO/ITUC sur la question des droits des travailleurs}

Le portrait idéal de Cuba qui apparaît dans le rapport de l'ICTU, et a fortiori dans le rapport de SIPTU de 2009, rejoint celui qui est fait par les associations civiles pro-cubaines, telles que Cuba Solidarity Campaign ${ }^{42}$. Il convient de souligner qu'il existe moins de voix dissonantes qui se lèvent pour protester contre le régime castriste au sein du syndicalisme irlandais que parmi les syndicats australiens par exemple, avec l'Australian Workers' Union (un syndicat rassemblant plus de 100000 adhérents) qui signa une lettre afin de manifester son refus de rencontrer l'ambassadeur cubain jusqu'à la fin attestée de la répression à l'encontre des syndicalistes cubains ${ }^{43}$. Les droits des travailleurs touchent au cœur des revendications syndicales, en particulier en ce qui concerne les négociations collectives et l'indépendance des syndicats. Or la fédération européenne de syndicats European Trade Union Congress (ETUC), la confédération internationale de syndicats

41. En 2017, une brigade de 47 jeunes syndicalistes du Royaume-Uni et d'Irlande participèrent à la $12^{\mathrm{e}}$ campagne de solidarité envers Cuba (Cuba Solidarity Campaign). Ils représentaient plusieurs syndicats britanniques et irlandais (Unite, UNISON, TSSA, GMB, RMT and CWU). Ils travaillèrent dans des fermes et participèrent à des conférences aux côtés de 286 membres des May Day brigades de 29 pays différents. Dans le bilan de leur voyage, ils précisèrent : "In addition to free university and medical school education that Cuba provides for its own citizens, the socialist island also offers free medical scholarships to young people from across the developing world. At the University Hospital in the delegation met with students from a wide range of countries including South Africa, Venezuela, Bolivia and Western Sahara. "Voir le site internet de l'association britannique Cuba Solidarity, "Record-breaking 47 young trade unionists participate May Day brigade in Cuba ", 26 mai 2017, [http:// www.cuba-solidarity.org.uk/news/article/3379/record-breaking-47-young-trade-unionists-participate-mayday-brigade-in-cuba], consulté le 27 février 2018.

42. Cuba Solidarity Campaign, "Trade unions: a Cuba Solidarity Campaign fact sheet ", 2015. Voir aussi Debra Evenson, "Workers in Cuba: unions and labour relations », Institute of Employment Rights, Londres, 2003, p..3-28.

43. La lettre, datée de janvier 2011, est signée de Paul Howes, le secrétaire national de l'Australian Workers' Union. Elle s'appuie notamment sur des rapports publiés par l'ILO et ITUC. Elle précise : "I would be happy to take up your offer to meet but this can, unfortunately, only happen once the Cuban government stops repressing independent trade unions and releases the many union leaders now in prison. " [http://www.labourstart.org/cuba/howes.pdf], consulté le 27 février 2018. 
International Trade Union Confederation (ITUC, qui par ailleurs n'a pas de syndicat affilié et basé à Cuba) et l'organisation internationale du travail International Labour Organization (ILO) ont tous fait part de leur réticence au sujet du régime castriste ${ }^{44}$.

Cuba est l'un des rares endroits au monde où le plus grand pourvoyeur d'emploi est l'État. Il paye les salaires et régule les horaires et les conditions de travail par publication de décrets. Dans son bilan annuel des violations des droits des syndicats sur l'île, l'ILO souligne le décalage entre ce qui est légal et ce qui est toléré en pratique par les autorités. En effet, le gouvernement cubain ne reconnait qu'une fédération nationale de syndicats, le Central de Trabajadores Cubanos (CTC). On retrouve les personnalités du CTC dans les plus hautes sphères de l'État. Selon l'ILO, le code du travail cubain, qui fut publié en 1985 et modifié en 2014, ne défend pas de véritable liberté d'association. Le gouvernement interdit les syndicats indépendants de la fédération nationale, bien qu'il n'y ait pas d'obligation légale pour ceux-ci de s'affilier au CTC ${ }^{45}$. Selon les autorités cubaines, citées par l'ILO: "Freedom of association, protected in Convention 87, does not translate into the false concept of 'trade union pluralism' imposed by the main centres of capitalist and imperial power ${ }^{46}$ ». L'ITUC souligne le fait qu'il n'y a pas de législation sur le droit de grève sur l'île et qu'en pratique :

No independent trade union activity is possible. Such organisations that do exist are unable to represent workers effectively. As they are not recognised, they cannot engage in collective bargaining or take strike action. Workers are not able to exercise their rights or to take part in peaceful marches or demonstrations in support of their demands. Workers are required to keep an eye on their colleagues and report any 'dissident' activity. Independent labour

44. ETUC dénonce toutefois l'embargo américain sur Cuba. Voir par exemple ETUC, «Executive committee Brussels, 1-2 June 2010 », (EC188/EN/7a), 2010, p. 11. En 2006, ETUC réaffirma cette position : «We fully support Cuba's sovereign rights. At the same time, we expect all our partners to respect international standards-on human rights; on trade union rights: freedom of association and collective bargaining; non-discrimination; no child labour; no forced labour. People should not be harassed or jailed for seeking to exercise those rights. The international community has set those standards. They should be universally applied. That is also why we unreservedly condemn the American blockade of Cuba, enforced now for forty years, and made even worse by the Helms-Burton Act of 1996. " Communiqué d'ETUC à Londres le 25 février 2006, disponible sur le site internet d'ETUC, [https://www. etuc.org/speeches/european-trade-union-solidarity-conference-cuba-and-latin-america\#.WpZ_p3yDPIU], consulté le 27 février 2018.

45. Rolando H. Castañeda et George Plinio Montalván, «El sistema laboral cubano y la irresponsabilidad social corporativa de los inversionistas extranjeros : puntos de vista de sindicalistasy periodistas independientes ", Cuba in transition, vol.19, Miami, 2009, p. 105.

46. International Labour Organization, « Rapport n 337 (Cuba) », juin 2005, [http://www.ilo.org/dyn/normlex/ en/f?p=NORMLEXPUB:50002:0::NO::P50002_COMPLAINT_TEXT_ID:2907759], consulté le 27 février 2018. 
activists are periodically arrested, harassed, threatened with prosecution and pressurised into going into exile ${ }^{47}$.

Quand elle était à Cuba en 2009, la délégation de SIPTU rencontra le CTC mais aucune question concernant la pluralité politique ne fut posée. Cette question fut en revanche soulevée lors d'une rencontre avec Oscar Martínez, directeur adjoint du département international du parti communiste cubain, en présence de Manuel Montero Bistilleiro du CTC, et la réponse reçue fit état du fait que Cuba était bel et bien une démocratie représentative : "There was an opportunity for all citizens to express their political opinion. If there were a multi-party system in operation they would be unable to get the current spend on Health and Education ${ }^{48}$." Le caractère discutable de l'argument invoqué par les autorités cubaines n'est pas commenté dans le rapport rédigé par SIPTU.

En 1958, la révolution de Castro fut caractérisée par son appel à une grève générale lancé à tous les travailleurs de l'île, mais les rapports successifs de l'ONG Human Rights Watch soulignent l'intimidation et les risques d'emprisonnement qui frappent les syndicalistes cubains ${ }^{49}$. Un tel paradoxe ne peut manquer de mettre en relief le décalage entre le discours des syndicats irlandais et la réalité de la condition des travailleurs sur l'île. Le mythe cubain qui apparaît dans le discours sur Cuba en Irlande est nourri de l'adhésion à certaines valeurs fondatrices du régime castriste comme l'anti-impérialisme. Il se fonde sur le souhait de croire à la possibilité de l'utopie socialiste, facilitée par la distance géographique entre les deux îles et l'exotisme de Cuba.

De manière plus concrète, les célébrations de 2009 permirent à l'ICTU et à certains syndicats irlandais, dont SIPTU, de réaffirmer leur engagement dans deux grandes campagnes liées à la " cause cubaine » : la demande de la fin de l'embargo américain sur l'île et la libération des « cinq de Miami ».

\section{Les syndicats irlandais en campagne pour Cuba}

Pour les syndicats irlandais, Cuba est un objet de discours, parfois un objet de fascination, mais c'est aussi une cause à défendre, un allié contre l'impérialisme de

47. International Trade Union Confederation, "Cuba: Annual Survey of Violations of Trade Union Rights », 2006. L'ITUC fit le même constat en 2009: "According to the ITUC figures, governments in at least nine countries - Burma, Burundi, China, Cuba, Iran, South Korea, Tunisia, Turkey and Zimbabwe-were responsible for imprisoning trade unionists on account of their legitimate activities in support of working people. " Dans International Trade Union Confederation, "Annual Survey of Violations of Trade Union Rights », 2009. Rapports disponibles sur le site internet de l'ITUC, [http://www.ituc-csi.org/ituc-annual-survey-of-trade-union?lang=fr], consulté le 27 février 2018.

48. SIPTU, "Report on visit to Cuba by the SIPTU Solidarity with Cuba Forum November 9th-18th, 2009 ", 2009, p. 13.

49. Human Rights Watch, "Cuba: Summary and recommendations », [https://www.hrw.org/reports/1999/cuba/ Cuba996-01.htm], 1999, consulté le 27 février 2018. 
la domination américaine à qui il convient d'apporter du soutien, dans le cadre de l'internationale socialiste. Un tel engagement s'incarna dans deux campagnes qui trouvèrent un nouvel élan dans les célébrations du cinquantenaire de la révolution en 2009.

\section{Sus à l'embargo américain sur Cuba}

En 1960-62, suite à la révolution cubaine, les États-Unis imposèrent un embargo économique, commercial et financier sur Cuba. Un contexte international très tendu prévalait alors, marqué par la guerre froide, avec la tentative d'invasion américaine par la baie des cochons en 1961 et la crise des missiles cubains en 1962. L'embargo fut par la suite renforcé par la loi Helms Burton de 1996, qui avait pour objectif de précipiter la chute de Castro. Vingt ans plus tard, Barack Obama prit la décision historique de l'alléger et de rétablir des relations diplomatiques avec le régime cubain. Cette politique fut remise en compte par son successeur Donald Trump, lequel revint en particulier sur le très symbolique visa touristique qui permettait aux citoyens américains de se rendre directement sur l'île ${ }^{50}$. Depuis 1992, l'Assemblée Générale des Nations Unis passe chaque année une résolution condamnant l'impact de l'embargo américain sur Cuba et soulignant que celui-ci va à l'encontre de la Charte des Nations Unis et des lois internationales ${ }^{51}$.

Pendant la conférence bisannuelle de l'ICTU en 2009, les syndicats Unite et Amalgamated Transport and General Workers Union présentèrent une motion $\left(\mathrm{n}^{\circ}\right.$ 68) consacrée aux questions internationales. Elle comprenait un volet sur Cuba, plus particulièrement sur la levée de l'embargo américain sur l'île : "To campaign in support of the people in Cuba and, in particular, to raise the demand that the US embargo and sanctions be lifted immediately without preconditions at every possible opportunity ${ }^{2}$. " Ce positionnement s'inscrivait dans la droite ligne de la création du Solidarity Forum with Cuba au sein du syndicat SIPTU en 2005. En effet, le groupe avait ratifié la décision de son homologue européen d'amplifier les actions jusqu'à ce que l'embargo soit levé. Dans cette optique, une levée de fonds, ainsi qu'un convoi humanitaire furent organisés avec différents syndicats étrangers en 2011. Lors de la conférence bisannuelle de l'ICTU, Margaret McKee, qui représentait le syndicat irlandais Unison, témoigna de l'initiative en

50. The Irish Times, "US government restricts travel to Cuba with new rules ", 8 novembre 2017.

51. Voir les résolutions 46/407, 47/19, 48/16, 49/9, 50/10, 51/17, 52/10, 53/4, 54/21, 55/20, 56/9, 57/11, 58/7, 59/11 et 60/12 de l'Assemblée Générale des Nations Unies. Sur la question des résolutions de l'ONU voir la publication de l'ICTU : «Embargo like NAMA each year », The Union Post, novembre 2009, p. 10.

52. Motion 68, ICTU, «Building Solidarity: Biennial delegate conference report, Tralee 7-10 July, 2009 », 2009, p. 39. 
ces termes: "we filled two ships with buses, ambulances, computers and even a train and delivered them through the US blockade ${ }^{33}$ ".

Après le nouvel élan donné en 2009 à la campagne des syndicats irlandais contre l'embargo américain sur Cuba, l'effort se poursuivit. En 2015, le journal syndicaliste cubain Trabajadores Daily (CTC) publia un article intitulé "Irish against the blockade ", signé de Orestes Eurgellés Mena ${ }^{54}$. Des représentants de Union of Industry, Professionals, Construction and Public Administration of Ireland vinrent réclamer à La Havane la fin de l'embargo lors d'une rencontre avec des cadres du Central de Trabajadores Cubanos. Des événements eurent régulièrement lieu en Irlande, comme "Mr Obama, Unblock the blockade ", organisé par Unite the Union en collaboration avec l'association civile Friends of Cuba ${ }^{55}$ en $2016^{56}$.

\section{Les cinq de Miami : Gerardo Hernández, Ramón Labañino, Fernando González, Antonio Guerrero, René González}

La seconde campagne dans laquelle les syndicats irlandais s'investirent en 2009 pour la cause cubaine fut la demande de la libération des cinq de Miami, dont le nom réveille le souvenir d'autres campagnes liées à l'histoire des Troubles en Irlande du Nord. Il fait référence à cinq citoyens cubains emprisonnés aux ÉtatsUnis après avoir été arrêtés par le FBI, le 12 septembre 1998. Ils furent condamnés, en 2001, à Miami, pour conspiration avec l'intention de mener des activités d'espionnage, de meurtre et autres activités illégales ${ }^{57}$. Leurs sentences, qui s'échelonnaient de 15 ans d'emprisonnement à la prison à vie, furent dénoncées comme injustes et de nature politique par des figures internationales, telles que l'Archevêque Desmond Tutu et l'ancien président américain Jimmy Carter. Les cinq hommes assurent qu'ils furent arrêtés alors qu'ils enquêtaient sur les activités de cellules terroristes anti-cubaines basées à Miami, lesquelles auraient mené des actions criminelles à Cuba comme la série d'explosions qui toucha des hôtels de La Havane en 1997, ainsi qu'un avion de ligne ${ }^{58}$.

53. ICTU, «Report of Proceedings of Biennial Delegate Conference », 2011, p. 103.

54. Orestes Eurgellés Mena (CTC), "Irish against the blockade », Trabajadores Daily, 16 novembre 2015.

55. Le groupe Friends of Cuba, ou Cuba Support Group fut créé en 1993 pour sensibiliser l'opinion publique à la question cubaine.

56. Unite the Union, «Mr Obama, Unblock the Blockade! Cuban Information Evening ", [https://www.facebook. com/events/1571971123110584/], consulté le 27 février 2018.

57. Les chefs d'accusation étaient les suivants: «conspiracy to commit murder, acting as an agent of a foreign government, and other illegal activities». Communiqué de presse de l'ICTU, "Congress president calls for immediate release of 'Cuban five' ", 12 septembre 2013, [https:/www.ictu.ie/press/2013/09/12/congress-president-callsfor-immediate-release-of-cuban-five], consulté le 27 février 2018.

58. 73 personnes décédèrent dans le crash d'un avion de ligne qui reliait la Barbade à la Jamaïque en 1976 (vol Cubana de aviación 455). Un touriste italien succomba dans l'explosion qui frappa l'un des trois hôtels de La Havane en septembre 1997. 
En 2009, John Douglas, secrétaire général de Mandate et futur président de l'ICTU (2013-2015), rencontra Teresita Trujillo, première ambassadrice de Cuba en Irlande, tout récemment nommée. Plus tard, en 2012, le Galway Council of Trade Unions invita Magali Llort, la mère de Fernando González, pour témoigner lors d'une rencontre destinée à informer et sensibiliser le public à la question des cinq de Miami59. En 2013, le même John Douglas réaffirma au nom de l'ICTU le soutien de plusieurs personnalités syndicales à la commission d'enquête dans l'affaire des cinq de $\mathrm{Miami}^{60}$ : "The entire process surrounding the conviction of the Cuban Five was dubious to say the least and, in the interests of justice, the US government must release these men immediately and allow them to return to their home country [...] They should be erecting statues to these men, not jailing them ${ }^{61}$ ». La même année une veillée fut organisée pour les cinq hommes au pied de la Spire, sur O'Connell Street, à Dublin. Les personnes présentes portaient un ruban jaune en hommage aux cinq de Miami et John Douglas adressa un discours au public.

La mobilisation des syndicats en faveur des cinq de Miami, qui prit un nouvel essor en 2009, atteignit son terme en 2014. En effet, René Gonzalez avait été libéré en 2011 après avoir terminé sa peine en prison ${ }^{62}$ et Fernando González fut libéré le 27 février 2014, suivi d'Antonio Guerrero, Ramón Labañino, et Gerardo Hernández le 17 décembre 2014. Ces libérations en série eurent lieu dans le cadre de la normalisation des relations avec Cuba, souhaitée par le gouvernement de Barack Obama. En signe de bonne volonté, Cuba libéra Alan Gross, travailleur humanitaire américain qui fut emprisonné pour avoir importé du matériel de télécommunication interdit sur l'île $e^{63}$. Les commentateurs s'accordèrent pour considérer la décision de Barack Obama comme étant courageuse, puisqu'elle fut âprement critiquée par la communauté cubaine installée en Floride, laquelle est farouchement opposée au régime de Castro. En effet, cette communauté cubaine en exil constitue un lobby important au sein de l'un des swing states et la libération des cinq de Miami intervint un peu plus d'un an seulement avant les élections présidentielles de 2016.

Le cinquantenaire de la révolution cubaine fut l'occasion pour l'ICTU et différents syndicats irlandais de réaffirmer leur solidarité avec le peuple cubain. Il permit de relancer les efforts engagés dans les deux campagnes principales, contre

59. "Galway Council of Trade Unions in Solidarity with the Cuban Five », [http://www.minrex.gob.cu/en/galwaycouncil-trade-unions-solidarity-cuban-five], consulté le 27 février 2018.

60. Celle-ci fut organisée en mars 2014, à Londres, par la Law Society en présence de l'un d'eux, René González, qui avait effectué ses quinze années de détention.

61. Communiqué de presse de l'ICTU, "Congress president calls for immediate release of 'Cuban five'», 12 septembre 2013, [https://www.ictu.ie/press/2013/09/12/congress-president-calls-for-immediate-release-of-cuban-five], consulté le 27 février 2018.

62. Damien Cave, "One of 'Cuban Five' Spies Is Released on Probation ", The New York Times, 7 octobre 2011. 63. "What's the new deal between the US and Cuba? ", Thejournal.ie, 18 décembre 2014. 
l'embargo américain et pour la libération des "cinq de Miami ", autour de l'organisation de lobbying politique, de levées de fond, de conférences et de veillées.

\section{Conclusion}

L'ambivalence avouée qui caractérise les relations de certaines fédérations internationales de travailleurs, telles que l'ILO ou l'ETUC/ITUC, avec Cuba, ne se retrouve pas chez l'ICTU, et, a fortiori, chez SIPTU. Cuba est célébrée par les syndicats irlandais comme un exemple de résistance contre la domination américaine, à travers la promotion de personnages emblématiques tels que José Martí et James Joseph O'Kelly, dont les parcours rapprochèrent l'histoire de l'île d'Irlande de celle de l'île de Cuba. De surcroît, le discours syndicaliste se nourrit de valeurs communes défendues par le régime castriste, telles que l'égalité des sexes, le droit à l'éducation ou encore à un système de santé égalitaire.

Le triple anniversaire de 2009, qui marqua à la fois le centenaire de la révolution cubaine, de la fondation de SIPTU et le cinquantenaire de la création de l'ICTU, permit de donner un nouvel élan aux campagnes contre l'embargo américain et pour la libération des cinq de Miami. D’un côté les syndicalistes voudraient croire à l'utopie socialiste, adhérer au mythe de la révolution cubaine contre l'impérialisme, de l'autre nul ne saurait ignorer les atteintes aux droits de l'homme qui y sont inquiétantes, en particulier concernant le droit des travailleurs et les intimidations subies par les syndicalistes locaux. Le malaise de certains syndicalistes irlandais transparaît entre les lignes des rapports de 2009, lesquels montrent que les délégations de syndicats sont emmenées autour de l'île pour visiter écoles et hôpitaux mais ne reçoivent pas de réponse convaincante concernant le droit des citoyens à quitter l'île ou le pluralisme politique.

L'histoire révolutionnaire de Cuba, fondée sur le rejet de l'Empire espagnol, fut marquée par un dialogue avec les idéologies socialistes et nationalistes, comme ce fut aussi le cas en Irlande. Par conséquent, aux côtés des syndicats, le parti républicain Sinn Féin prête une attention toute particulière à l'île. Un monument fut dévoilé à La Havane en 2001 par Gerry Adams, alors leader du Sinn Féin, pour honorer la mémoire des républicains irlandais qui perdirent la vie lors de grèves de la faim en Irlande du Nord dans les années $1980^{64}$. La récente reconnaissance par Hermes Herrera Hernández, ambassadeur cubain à Dublin, qu'à l'instar de Fidel Castro lui-même, il avait soutenu l'action de l'IRA, a fait couler beaucoup d'encre $^{65}$. Castro décrivit les grèves de la faim des républicains en Irlande du Nord comme " le geste le plus émouvant de sacrifice, d'altruisme et de courage " et les

64. Gerry Adams, "Cuba and Ireland: Solidarity in struggle ", An Phoblacht, 20 décembre 2001.

65. Cate McCurry, «I backed Castro's IRA stance: Cuban ambassador to Republic », The Belfast Telegraph, 16 février 2017. 
grévistes comme ayant gagné « le respect et l'admiration du monde ${ }^{66}$ ». On ne peut qu'espérer qu'aucun autre prisonnier de conscience ne mourra dans les geôles cubaines suite à une grève de la faim, comme ce fut le cas de l'activiste Orlando Zapata Tamayo en $2010^{67}$. La poursuite ou non de la politique de détente, initiée par Obama en 2013-2014, et actuellement remise en cause par le gouvernement de Trump, aura un impact fondamental sur l'avenir de Cuba, son inscription dans les réseaux internationaux de syndicats et l'évolution des droits des travailleurs et des opposants politiques sur l'île.

66. Les termes précis sont " most moving gesture of sacrifice, selflessness and courage " et " earned the respect and admiration of the world ». Cate McCurry, "I backed Castro's IRA stance: Cuban ambassador to Republic ", The Belfast Telegraph, 16 février 2017.

67. Raúl Castro a affirmé regretter la mort d’Orlando Zapata Tamayo, décédé en prison en 2010 après 85 jours de grève de la faim. 